\title{
Identification of Sources of Resistance to Xanthomonas campestris pv. campestris in Brassica napus Crops
}

\author{
M. Lema, P. Soengas, P. Velasco, M. Francisco, and M. E. Cartea, Department of Plant Genetics, Misión Biológica de Galicia \\ (CSIC), E-36080 Pontevedra, Spain
}

\begin{abstract}
Lema, M., Soengas, P., Velasco, P., Francisco, M., and Cartea, M. E. 2011. Identification of sources of resistance to Xanthomonas campestris pv. campestris in Brassica napus crops. Plant Dis. 95:292-297.

Black rot, caused by Xanthomonas campestris pv. campestris, is one of the most important diseases affecting Brassica crops worldwide. Nine races have been differentiated in $X$. campestris pv. campestris, with races 1 and 4 being the most virulent and widespread. The objective of this work was to identify sources of resistance to races 1 and 4 of $X$. campestris pv. campestris in different Brassica napus crops, mainly in the underexplored pabularia group. Seventy-six accessions belonging to four $B$. napus groups were screened for resistance to two $X$. campestris pv. campestris races (1 and 4). The strain of race 1 used in this study was more virulent on the tested materials than the strain of race 4. No race-specific resistance was found to race 1. Most cultivars were

susceptible except Russian kale, from the pabularia group, which showed some resistant plants and some other accessions with some partially resistant plants. High levels of race-specific resistance to race 4 were found in the pabularia group, and great variability within accessions was identified. Three improved cultivars (Ragged Jack kale, Friese Gele, and Valle del Oro) and four landraces (Russian kale, MBG-BRS0037, MBG-BRS0041, and MBG-BRS0131) showed plants with some degree of resistance to both races, which may indicate that race-nonspecific resistance is involved. These accessions could be directly used in breeding programs, either as improved cultivars or as donors of race-specific resistance to other Brassica cultivars.
\end{abstract}

Black rot is one of the most devastating diseases in Brassica crops and has worldwide distribution. This bacterial disease is caused by Xanthomonas campestris pv. campestris (Pammel) Dowson. The seedborne pathogen can survive in crop debris or crucifer weeds and is especially damaging in vegetable Brassica crops, mainly in warm and humid climates. In coastal temperate areas of Europe, the disease is not able to kill the plant (35) but it makes V-shaped necrotic lesions on leaf margins, which decrease the quality on the fresh market. Moreover, this disease weakens plant tissues, potentially leading to infection from other pathogens. Infected seed are the primary source of inoculum. When optimal conditions for bacterial development are not present, no symptoms are expressed; however, the pathogen can remain in the vascular system of these asymptomatic plants. Symptoms may be expressed on a susceptible cultivar when conditions favor disease development.

In Spain, which is an important Brassica spp. producer and consumer, the presence of $X$. campestris pv. campestris was described in the 1970 s by Urquijo et al. (29) and later by Ortega and López (18). Urquijo et al. (29) determined the presence of $X$. campestris pv. campestris in Brassica napus, B. oleracea, and B. rapa crops from northwest, central, and eastern Spain based on the symptoms of the disease in infected plants. Ortega and López (18) identified $X$. campestris pv. campestris from different $B$. oleracea crops (broccoli, Brussels sprout, cabbage, and cauliflower) from the Mediterranean coast of Spain based on morphological and biochemical characteristics of the pathogen and in a pathogenicity test (isolation, growth of the bacterium in pure culture, and reinoculation of susceptible host plants). In northwestern Spain, black rot has been recently identified in several Brassica crops (14) and a study referred to the incidence of $X$. campestris pv. campestris races is being carried out (unpublished data). According to our pre-

Corresponding author: M. Lema, E-mail: mlema@mbg.cesga.es

Accepted for publication 22 October 2010.

doi:10.1094/PDIS-06-10-0428

(C) 2011 The American Phytopathological Society liminary results, race 4 is the most prevalent in this area, followed by races 1 and 6 , although it is possible that other races are also present (races 2, 7, and 9). In this region, the production is mainly by small growers who do not use healthy plant material or diseasefree seed; consequently, the pathogen can spread rapidly. No studies involving either the pathogen or screens for resistance in this area have been conducted.

Williams (34) illustrated a number of control measures against this disease; specifically, the use of resistant cultivars and the exploration of new sources of resistance. Currently, disease management is still complicated and limited sources of resistance are available. Recently, the search for new sources of resistance has focused on race-specific resistance since the existence of six races of the pathogen was described by Vicente et al. (31). The authors analyzed $164 X$. campestris pv. campestris isolates from various origins worldwide and established a Brassica differential series, thus modifying previous classifications by Kamoun et al. (13) and Ignatov et al. (11). The interaction between differential cultivars and $X$. campestris pv. campestris races is explained by using a gene-for-gene model. In addition to monogenic race-specific resistance $(4,8,31,32)$, a quantitative race-nonspecific resistance has been described $(3,7,23,25,32)$. Recently, Fargier and Manceau (5) analyzed 47 representative strains of $X$. campestris and added three new races (races 7, 8, and 9) to the six races described by Vicente et al. (31). It is recognized that races 1 and 4 are the most virulent and widespread, accounting for most black rot cases around the world $(6,31)$.

Sources of resistance to X. campestris pv. campestris in Brassica genomes have been examined by Bain (2), Hunter et al. (10), Guo et al. (8), Nogueira and Dias (17), Ribeiro and Dias (19), Westman et al. (33), Taylor et al. (25), Massomo et al. (16), Tonguç and Griffiths (28), Jensen et al. (12), and Griffiths et al. (6). To the authors' knowledge, the use of resistant cultivars has only had limited success, and available sources with useful levels of resistance are scarce. Moreover, most of this work focused in cabbage $(B . o l$ eracea var. capitata) due to its economical value, while the search for resistance in B. napus has been more restricted. Guo et al. (8) identified two highly resistant accessions of B. napus (plant introduction [PI] 199947 and PI 199949) but they were reidentified later as $B$. carinata (25). In this study, several crops from the Brassica- 
ceae family were evaluated, including 38 accessions of B. napus, and resistance to race 1 was not found. Recently, Griffiths et al. (6) evaluated 558 B. napus accessions, and incomplete resistance to $X$. campestris pv. campestris was found in two accessions from South Korea (PI 469733 and PI 469828).

From the taxonomic point of view, B. napus can be divided into four groups according to the edible part of the plant (seed, roots, and leaves) and their main end use (seed oil production and fresh consumption of vegetable parts; Table 1). Four classification groups are as follows: B. napus L. napobrassica (L.) Reichb. group (synonymous with B. napus L. rapifera (Metzg) group), which includes rutabaga or swede; $B$. napus L. napus group, which includes forage and fodder rape; B. napus L. oleifera (DC.) group, which includes oilseed rape; and, finally, B. napus pabularia (DC.) Reichb. group, which includes leaf rape, rape kale, Siberian kale, and Hanover salad (Table 1). Crops belonging to this last group are popular for human consumption in northwestern Spain and northern Portugal, where they are known as "nabicol" and "couvenabiça", respectively $(20,22)$. Black rot can be a serious problem in leafy-green vegetable crops belonging to this last group, where leaves must be completely free of any lesion.

Specific resistance to races 1 or 4 has been reported in $B$. carinata, B. juncea, B. nigra, B. rapa, and B. napus $(6,8,25,27,28)$. Within $B$. napus species, resistance has been only found in rapeseed (8), in one couve-nabiça cultivar (25), and in two accessions with unknown use collected from South Korea (6). However, an evaluation including the different groups from B. napus species has not been performed thus far, and breeding for $X$. campestris $\mathrm{pv}$. campestris resistance has not been undertaken in vegetable crops of $B$. napus. Therefore, the objective of this work was to identify sources of resistance to races 1 and 4 of $X$. campestris pv. campestris in different $B$. napus crops, mainly in the underexplored pabularia group. The relationship between $X$. campestris pv. campestris resistance and the different end-use of $B$. napus crops is also discussed.

\section{Materials and Methods}

Plant accessions. In total, 76 B. napus accessions (47 landraces and 29 commercial and improved cultivars) from different geographic origins were evaluated (Table 2). Accessions were divided based on the end-use of the material following the European Brassica database nomenclature (http://documents.plant.wur.nl/cgn/pgr/ brasedb/) into four sets: (i) the napus group included 10 accessions used as forage crops, (ii) the oleifera group included 9 accessions of spring and winter rapeseed used as oilseed crops, (iii) the napobrassica group included 9 accessions used as root crops, and (iv) the pabularia group included 48 accessions, mostly from Spain, used as leafy-green vegetable crops.

A differential resistance series established by Vicente (31), including cv. Wirosa $F_{1}$ (B. oleracea, susceptible to both races), doubled-haploid line $\mathrm{S} \times \mathrm{D} 1$ (B. oleracea, susceptible to both races), doubled-haploid line Cob60 (B. napus, derived from line 14R of cv. Cobra, resistant to race 4), cv. Seven Top Turnip (B. rapa, resistant to race 4 although variable), inbred line PIC1 (B. carinata, a selection of PI 199947, resistant to races 1 and 4), and doubledhaploid line FBLM2 (B. juncea, derived from Florida Broad Leaf Mustard, resistant to races 1 and 4), was used (Table 2).

Four pots containing four plants were sown by accession. Pots were watered by subirrigation using a flood-bench system. Water was delivered from below and was allowed to flood the bench for $30 \mathrm{~min}$, after which excess water was drained. This process was repeated twice per week.
Pathogen isolates. Bacterial isolates of race 1 type strain HRI3811, synonymous with PHW1205 (collected on B. oleracea in the United States) and race 4 type strain HRI1279A (collected from B. oleracea var. capitata in the United Kingdom), typed by Vicente et al. (31), and provided by Warwick HRI, Wellesbourne, UK, were used. Bacterial cultures were grown in bacterial screening media 523 at $30^{\circ} \mathrm{C}$ during $48 \mathrm{~h}$ prior to inoculation. Bacterial culture was diluted in sterile tap water until suspension reached a density of $5 \times 10^{8} \mathrm{cfu} \mathrm{ml}^{-1}$. This concentration corresponded to a spectrophotometer reading of 0.5 at a wavelength of $600 \mathrm{~nm}$.

Inoculations and disease resistance assays. Sixteen plants per accession were inoculated 4 to 5 weeks after planting. Two different leaves of the same plant were used for inoculations. The third leaf was inoculated with race 1 and the fourth leaf with race 4 . After inoculation, greenhouse conditions were maintained at $14 \mathrm{~h}$ of light, mean temperature $24^{\circ} \mathrm{C}$ at night and $28^{\circ} \mathrm{C}$ during the day and relative humidity between 80 and $90 \%$. Multiple needles were used for inoculation according to Lema-Márquez et al. (15). Plants were rated for disease reaction twice, at 14 and 21 days after inoculation. Disease evaluations were made on a visual 1-to-9 rating scale based on the relative lesion size, where $1=$ no visible symptoms; 3 = an isolated, diffuse chlorotic zone around necrotic lesions with blackened veins of $<25 \%$ of the inoculated area; $5=$ chlorotic zones around necrotic lesions with blackened veins occupying $<50 \%$ of the inoculated area; $7=$ complete chlorosis, with black veins and usually necrotic centers of the inoculated area and chlorotic or necrotic lesions extending beyond the inoculated area; and $9=$ severely diseased, typical leaf edge damages, with large Vshaped chlorotic lesions presenting areas with blackened veins. For each inoculated leaf, two disease scores were taken (14 and 21 days post inoculation). Accessions with mean disease scores from 1 to 3 were considered resistant, 4 to 6 were partially or moderately resistant, and 7 to 9 were considered susceptible.

Statistical analyses. Analyses of variance were performed for disease score and were combined across races by using the general linear model procedure of SAS (21). Accessions and races were considered as fixed effects whereas replications (plants within accessions) were considered as a random factor. Comparisons of means were performed for each trait by using Fisher's protected least significant difference at the 0.05 level of probability (24). The sums of squares for accessions were orthogonally divided into groups according to their final use.

\section{Results}

The combined analysis of variance across races showed that accessions and races were significantly different $(P \leq 0.05)$ for disease scores (data not shown). The race-accession interaction was also significant, indicating that accessions performed distinctly against two races involved in race-specific resistance. Because this interaction was significant, individual analyses of variance for each race were conducted (data not shown). Therefore, results will be discussed separately for each race. The ranges of resistance responses and mean disease score of 76 accessions of B. napus inoculated with isolates of two $X$. campestris pv. campestris races (races 1 and 4) shown by individual accessions are in Table 2. The results of the resistance tests by taxonomic group are shown in Table 3.

Race 1. In the analyses of variance, significant differences were found for disease score among the 76 B. napus accessions evaluated, among the four taxonomic groups defined a priori (napobrassica, napus, oleifera, and pabularia) and among accessions within each taxonomic group. No race-specific resistance to race 1 was

Table 1. Description of the main taxonomical groups included in Brassica napus

\begin{tabular}{llccc}
\hline Group & & Part used & End use & Consumption \\
\hline napobrassica or rapifera & Rutabaga or swede & Root & Fresh & Animal and human \\
napus & Forage or fodder rape & Leaf & Fresh & Animal \\
oleifera & Oilseed rape or rape & Seed & Oil & Human \\
pabularia & Leaf rape, rape kale, Siberian kale, and Hanover salad & Leaf & Fresh & Animal and human \\
\hline
\end{tabular}


found in the genotypes evaluated. Accessions from the napus group were slightly different from other groups, although all of them were susceptible based on their values for mean disease score (Table 3). Russian kale, a rape kale cultivar, included into the pabularia group, showed some degree of resistance to this race, having $25 \%$ resistant plants. This cultivar differed significantly from all other accessions evaluated and had the lowest mean disease score (5.3), ranging from 2 to 9 (Table 2). Three cultivarsFriese Gele (a commercial rutabaga), Ragged Jack kale (an improved forage rape), and Valle del Oro (a commercial winter oilseed rape)-along with three nabicol landraces from north- western Spain (MBG-BRS0037, MBG-BRS0041, and MBGBRS0131, included into the pabularia group) presented some plants with moderate resistance with disease scores ranging from 5 to 6 (Table 2 ).

Race 4. The analyses of variance indicated differences in disease score according to accessions, groups, and accessions within each corresponding group. Differences among groups were greater than those found for race 1. A high level of race-specific resistance to race 4 was found in the pabularia group. This group had the highest number of accessions, including more than one resistant plant (42 of 48), and showed the lowest mean disease score (3.4), being

Table 2. Description and percentage of resistant plants and mean and range of disease score for Xanthomonas campestris pv. campestris races 1 and 4 for 76 Brassica napus accessions for resistance to Xanthomonas campestris pv. campestris conducted in Pontevedra (Spain) in 2008

\begin{tabular}{|c|c|c|c|c|c|c|c|c|c|c|}
\hline \multirow[b]{3}{*}{ Accession no. ${ }^{t}$} & \multirow[b]{3}{*}{ Status $^{u}$} & \multirow[b]{3}{*}{ Accession name } & \multirow[b]{3}{*}{ Origin } & \multirow[b]{3}{*}{ Source $^{v}$} & & & \multicolumn{4}{|c|}{ Disease score $(1-9)^{\mathrm{s}}$} \\
\hline & & & & & \multicolumn{2}{|c|}{ Resistant (\%) } & \multicolumn{2}{|c|}{ Race 1} & \multicolumn{2}{|c|}{ Race 4} \\
\hline & & & & & Race 1 & Race 4 & Range & Mean & Range & Mean \\
\hline \multicolumn{11}{|c|}{ Differential accessions } \\
\hline BE0116 & $\mathrm{AC}$ & $\mathrm{S} \times \mathrm{D}_{1}($ B. oleracea $)$ & United Kingdom & WHRI & 0 & 0 & 9 & 9.0 & $7-9$ & 8.3 \\
\hline BE0117 & $\mathrm{C}$ & Seven Top Turnip (B. rapa) & United Kingdom & WHRI & 0 & 82 & 9 & 9.0 & $1-9$ & 2.6 \\
\hline BE0118 & $\mathrm{AC}$ & Cob60 (B. napus) & United Kingdom & WHRI & 0 & 100 & 9 & 9.0 & $1-3$ & 1.8 \\
\hline BE0119 & $\mathrm{AC}$ & FBLM2 (B. juncea) & United Kingdom & WHRI & 100 & 100 & $1-2$ & 1.1 & $1-2$ & 1.2 \\
\hline BE0120 & $\mathrm{AC}$ & PIC 1 (B. carinata) & United Kingdom & WHRI & 100 & 100 & 1 & 1.0 & $1-3$ & 1.1 \\
\hline BE0121 & $\mathrm{C}$ & Wirosa $\mathrm{F}_{1}($ B. oleracea $)$ & Spain & Germisem & 0 & 0 & 9 & 9.0 & 9 & 9.0 \\
\hline \multicolumn{11}{|l|}{ Forage crops ${ }^{\mathrm{w}}$} \\
\hline BE0051 & $\mathrm{C}$ & Colza Rocalba & Spain & MBG & 0 & 70 & $7-8$ & 7.2 & $1-7$ & 3.2 \\
\hline BE0130 & $\mathrm{AC}$ & Lair & United Kingdom & CGN & 0 & 73 & $7-9$ & 8.6 & $1-7$ & 3.2 \\
\hline BE0132 & $\mathrm{AC}$ & $\mathrm{SjG}(6)$ & The Netherlands & CGN & 0 & 56 & $7-9$ & 8.5 & $1-8$ & 3.6 \\
\hline BE0128 & $\mathrm{AC}$ & Zls (4) & The Netherlands & CGN & 0 & 23 & $8-9$ & 8.9 & $2-7$ & 4.7 \\
\hline BE0131 & $\mathrm{AC}$ & O.W. (1) & The Netherlands & CGN & 0 & 39 & $7-9$ & 8.6 & $2-9$ & 4.8 \\
\hline BE0055 & $\mathrm{AC}$ & Mara & The Netherlands & CGN & 0 & 26 & $7-9$ & 8.5 & $2-8$ & 4.9 \\
\hline BE0054 & $\mathrm{AC}$ & Vysokopol'skij 12 & Russia & CGN & 0 & 13 & $7-9$ & 8.3 & $2-9$ & 6.3 \\
\hline BE0053 & $\mathrm{AC}$ & Ragged Jack kale & New Zealand & CGN & 0 & 12 & $6-9$ & 7.9 & $2-9$ & 7.2 \\
\hline BE0057 & $\mathrm{AC}$ & Potem & France & CGN & 0 & 8 & 7-9 & 8.7 & $2-9$ & 7.8 \\
\hline BE0129 & $\mathrm{AC}$ & Ramon & The Netherlands & CGN & 0 & 0 & 9-9 & 9.0 & $7-9$ & 8.9 \\
\hline Mean & & $\ldots$ & $\ldots$ & $\ldots$ & $\ldots$ & $\ldots$ & $\ldots$ & 8.4 & $\ldots$ & 5.4 \\
\hline $\mathrm{CV}$ & & $\ldots$ & $\ldots$ & $\ldots$ & $\ldots$ & $\ldots$ & $\ldots$ & 8.6 & $\ldots$ & 36.1 \\
\hline $\operatorname{LSD}(0.05)$ & & $\ldots$ & $\ldots$ & $\ldots$ & $\ldots$ & $\ldots$ & $\ldots$ & 0.5 & $\ldots$ & 1.3 \\
\hline \multicolumn{11}{|c|}{ Leafy vegetable crops $\mathrm{x}^{\mathrm{x}}$} \\
\hline MBG-BRS0092 & $\mathrm{L}$ & Nabicol & Spain & MBG & 0 & 100 & $7-9$ & 8.7 & $1-2$ & 1.2 \\
\hline MBG-BRS0085 & $\mathrm{L}$ & Nabicol & Spain & MBG & 0 & 100 & $7-9$ & 8.7 & $1-3$ & 1.3 \\
\hline MBG-BRS0087 & $\mathrm{L}$ & Nabicol & Spain & MBG & 0 & 100 & $7-9$ & 8.9 & $1-2$ & 1.3 \\
\hline MBG-BRS0088 & $\mathrm{L}$ & Nabicol & Spain & MBG & 0 & 100 & $7-9$ & 8.8 & $1-2$ & 1.4 \\
\hline MBG-BRS0105 & $\mathrm{L}$ & Nabicol & Spain & MBG & 0 & 100 & 9 & 9.0 & $1-3$ & 1.4 \\
\hline MBG-BRS0107 & $\mathrm{L}$ & Nabicol & Spain & MBG & 0 & 100 & $7-9$ & 8.8 & $1-3$ & 1.4 \\
\hline MBG-BRS0073 & $\mathrm{L}$ & Nabicol & Spain & MBG & 0 & 100 & $7-9$ & 8.0 & $1-3$ & 1.5 \\
\hline MBG-BRS0543 & $\mathrm{L}$ & Nabicol & Spain & MBG & 0 & 100 & $7-9$ & 8.4 & $1-3$ & 1.5 \\
\hline MBG-BRS0028 & $\mathrm{L}$ & Nabicol & Spain & MBG & 0 & 100 & 9 & 9.0 & $1-2$ & 1.6 \\
\hline MBG-BRS0090 & $\mathrm{L}$ & Nabicol & Spain & MBG & 0 & 100 & 9 & 9.0 & $1-3$ & 1.7 \\
\hline MBG-BRS0044 & $\mathrm{L}$ & Nabicol & Spain & MBG & 0 & 100 & 9 & 9.0 & $1-3$ & 1.7 \\
\hline MBG-BRS0034 & $\mathrm{L}$ & Nabicol & Spain & MBG & 0 & 93 & $7-9$ & 8.4 & $1-4$ & 1.8 \\
\hline MBG-BRS0041 & $\mathrm{L}$ & Nabicol & Spain & MBG & 0 & 100 & $5-9$ & 8.6 & $1-3$ & 1.8 \\
\hline MBG-BRS0110 & $\mathrm{L}$ & Nabicol & Spain & MBG & 0 & 100 & $7-9$ & 8.2 & $1-3$ & 1.8 \\
\hline MBG-BRS0113 & $\mathrm{L}$ & Nabicol & Spain & MBG & 0 & 100 & $7-9$ & 8.9 & $1-3$ & 1.8 \\
\hline MBG-BRS0356 & $\mathrm{L}$ & Nabicol & Spain & MBG & 0 & 100 & 9 & 9.0 & $1-3$ & 1.8 \\
\hline MBG-BRS0378 & $\mathrm{L}$ & Nabicol & Spain & MBG & 0 & 100 & 9 & 9.0 & $1-3$ & 1.9 \\
\hline MBG-BRS0054 & $\mathrm{L}$ & Nabicol & Spain & MBG & 0 & 100 & 9 & 9.0 & $1-3$ & 2.1 \\
\hline MBG-BRS0056 & $\mathrm{L}$ & Nabicol & Spain & MBG & 0 & 93 & $7-9$ & 8.2 & $1-5$ & 2.1 \\
\hline MBG-BRS0048 & $\mathrm{L}$ & Nabicol & Spain & MBG & 0 & 88 & 9 & 9.0 & $1-5$ & 2.2 \\
\hline MBG-BRS0065 & $\mathrm{L}$ & Nabicol & Spain & MBG & 0 & 100 & $8-9$ & 8.9 & $1-3$ & 2.2 \\
\hline MBG-BRS0039 & $\mathrm{L}$ & Nabicol & Spain & MBG & 0 & 94 & 9 & 9.0 & $1-4$ & 2.3 \\
\hline \multirow[t]{2}{*}{ MBG-BRS0014 } & $\mathrm{L}$ & Nabicol & Spain & MBG & 0 & 100 & $7-9$ & 8.9 & $1-3$ & 2.4 \\
\hline & & & & & & & & \multicolumn{3}{|c|}{ (continued on next page) } \\
\hline
\end{tabular}

\footnotetext{
${ }^{\mathrm{s}}$ Disease score based on a rating scale from 1 to $9(1=$ plants without lesions and $9=$ severely diseased plants $)$, where 1 to $3=$ resistant, 4 to $6=$ moderately or partially resistant, and 7 to $9=$ susceptible. Range indicates minimum and maximum disease score.

${ }^{\mathrm{t}} \mathrm{CV}=$ coefficient of variation, LSD $=$ least significant difference among accessions within groups, and LSD all $=$ LSD among all accessions.

" $\mathrm{AC}=$ advanced or improved cultivar, $\mathrm{C}=$ commercial cultivar, and $\mathrm{L}=$ traditional or local cultivar or landrace.

${ }^{\vee}$ Seed sources: CGN $=$ Center for Genetic Resources, Wageningen, The Netherlands; COMAV = Centro de Conservación y Mejora de la Agrodiversidad Valenciana, Valencia, Spain; WHRI = Warwick - Horticulture Research International, Wellesbourne, UK; MBG = Misión Biológica de Galicia $($ CSIC), Pontevedra, Spain.

${ }^{\mathrm{w}}$ Fodder or forage rape $=$ napus group.

${ }^{x}$ Couve-nabiça, Hanover kale, leaf-rape, nabicol, rape kale, or Siberian kale = pabularia group.

y Oilseed rape or rapeseed = oleifera group.

${ }^{\mathrm{z}}$ Rutabaga or swede $=$ napobrassica or rapifera group.
} 
significantly lower than those observed in napus (5.4), oleifera (6.2), and napobrassica (8.0) groups (Table 3).

Based on the mean disease score of each accession, leafy vegetable crops showed the highest percentage of resistant accessions with respect to the total accessions evaluated within each group (73\%), followed by forage (20\%), oilseed $(11 \%)$, and root vegetable $(0 \%)$ crops (Table 2). Approximately $50 \%$ of total accessions were resistant to race 4 , having a mean disease score less than 4 on a 1-to-9 rating scale (Table 2). Of these, 19 accessions, all belonging to pabularia group, were uniformly resistant. In the four groups, some accessions classified as susceptible or as moderately resistant were highly variable. In fact, a high proportion of acces- sions evaluated (40 of 76) produced variable resistance reactions, including both resistant and susceptible plants (Table 2).

In the pabularia group, nabicol accessions named MBGBRS0028, MBG-BRS0087, MBG-BRS0088, and MBG-BRS0092 were more resistant to race 4 , with all plants having disease scores between 1 and 2 (Table 2). Within the same group, the rape kale cv. Russian kale, found to be partially resistant to race 1 , can also be classified as resistant to race 4 based on its disease score, although levels of resistance were highly variable. Three commercial cultivars included in this group were also resistant or moderately resistant to race 4, although resistance was highly variable. Apart from the pabularia group, two napus cultivars (colza Rocalba and Lair)

Table 2. (continued from preceding page)

\begin{tabular}{|c|c|c|c|c|c|c|c|c|c|c|}
\hline \multirow[b]{3}{*}{ Accession no. ${ }^{t}$} & \multirow[b]{3}{*}{ Status ${ }^{u}$} & \multirow[b]{3}{*}{ Accession name } & \multirow[b]{3}{*}{ Origin } & \multirow[b]{3}{*}{ Source $^{v}$} & & & \multicolumn{4}{|c|}{ Disease score $(1-9)^{\mathrm{s}}$} \\
\hline & & & & & \multicolumn{2}{|c|}{ Resistant (\%) } & \multicolumn{2}{|c|}{ Race 1} & \multicolumn{2}{|c|}{ Race 4} \\
\hline & & & & & Race 1 & $\overline{\text { Race } 4}$ & Range & Mean & Range & Mean \\
\hline MBG-BRS0068 & $\mathrm{L}$ & Nabicol & Spain & MBG & 0 & 92 & 9 & 9.0 & $1-9$ & 2.5 \\
\hline MBG-BRS0434 & $\mathrm{L}$ & Nabicol & Spain & MBG & 0 & 93 & $7-9$ & 8.3 & $2-7$ & 2.8 \\
\hline MBG-BRS0035 & $\mathrm{L}$ & Nabicol & Spain & MBG & 0 & 83 & $8-9$ & 8.9 & $1-8$ & 3.1 \\
\hline MBG-BRS0346 & $\mathrm{L}$ & Nabicol & Spain & MBG & 0 & 82 & 9 & 9.0 & $1-7$ & 3.1 \\
\hline MBG-BRS0388 & $\mathrm{C}$ & Couve-nabiça & Portugal & MBG & 0 & 94 & 9 & 9.0 & $2-9$ & 3.1 \\
\hline MBG-BRS0423 & $\mathrm{L}$ & Nabicol & Spain & MBG & 0 & 75 & $7-9$ & 7.5 & $2-4$ & 3.2 \\
\hline MBG-BRS0542 & $\mathrm{L}$ & Nabicol & Spain & MBG & 0 & 71 & $7-9$ & 8.2 & $2-7$ & 3.2 \\
\hline MBG-BRS0582 & $\mathrm{L}$ & Nabicol & Spain & MBG & 0 & 74 & $7-9$ & 8.8 & $1-9$ & 3.2 \\
\hline MBG-BRS0373 & $\mathrm{C}$ & Couve-nabiça & Portugal & MBG & 0 & 81 & 9 & 9.0 & $2-8$ & 3.3 \\
\hline MBG-BRS0029 & $\mathrm{L}$ & Nabicol & Spain & MBG & 0 & 75 & $7-9$ & 8.7 & $1-9$ & 3.4 \\
\hline BE0002 & $\mathrm{C}$ & Nabicol Rocalba & Spain & MBG & 0 & 43 & $7-9$ & 8.6 & $2-7$ & 3.5 \\
\hline BE0085 & $\mathrm{L}$ & Russian kale & United Kingdom & WHRI & 25 & 50 & $2-9$ & 5.3 & $2-7$ & 3.5 \\
\hline MBG-BRS0541 & $\mathrm{L}$ & Nabicol & Spain & MBG & 0 & 75 & 9 & 9.0 & $2-7$ & 3.9 \\
\hline MBG-BRS0374 & $\mathrm{L}$ & Nabicol & Spain & MBG & 0 & 60 & 9 & 9.0 & $1-9$ & 4.1 \\
\hline MBG-BRS0134 & $\mathrm{L}$ & Nabicol & Spain & MBG & 0 & 47 & 9 & 9.0 & $2-7$ & 4.5 \\
\hline MBG-BRS0061 & $\mathrm{L}$ & Nabicol & Spain & MBG & 0 & 38 & $7-9$ & 8.2 & $1-8$ & 5.2 \\
\hline MBG-BRS0037 & $\mathrm{L}$ & Nabicol & Spain & MBG & 0 & 33 & $6-9$ & 8.1 & $2-9$ & 5.9 \\
\hline MBG-BRS0131 & $\mathrm{L}$ & Nabicol & Spain & MBG & 0 & 33 & $5-9$ & 7.8 & $2-9$ & 5.9 \\
\hline MBG-BRS0337 & $\mathrm{L}$ & Nabicol & Spain & MBG & 0 & 13 & 9 & 9.0 & $2-9$ & 6.6 \\
\hline MBG-BRS0465 & $\mathrm{L}$ & Nabicol & Spain & MBG & 0 & 7 & $7-9$ & 7.9 & $2-9$ & 6.9 \\
\hline MBG-BRS0333 & $\mathrm{L}$ & Nabicol & Spain & MBG & 0 & 0 & 9 & 9.0 & $7-9$ & 7.5 \\
\hline MBG-BRS0329 & $\mathrm{L}$ & Nabicol & Spain & MBG & 0 & 7 & 9 & 9.0 & $3-9$ & 8.1 \\
\hline MBG-BRS0079 & $\mathrm{L}$ & Nabicol & Spain & MBG & 0 & 0 & $7-9$ & 8.9 & $7-9$ & 8.2 \\
\hline MBG-BRS0583 & $\mathrm{L}$ & Nabicol & Spain & MBG & 0 & 0 & 9 & 9.0 & $7-9$ & 8.5 \\
\hline MBG-BRS0063 & $\mathrm{L}$ & Nabicol & Spain & MBG & 0 & 0 & 9 & 9.0 & $7-9$ & 8.8 \\
\hline Mean & $\ldots$ & $\ldots$ & $\ldots$ & $\ldots$ & $\ldots$ & $\ldots$ & $\ldots$ & 8.7 & $\ldots$ & 3.4 \\
\hline $\mathrm{CV}$ & $\ldots$ & $\ldots$ & $\ldots$ & $\ldots$ & $\ldots$ & $\ldots$ & $\ldots$ & 7.8 & $\ldots$ & 44.8 \\
\hline LSD (0.05) & $\ldots$ & $\ldots$ & $\ldots$ & $\ldots$ & $\ldots$ & $\ldots$ & $\ldots$ & 0.5 & $\ldots$ & 1.1 \\
\hline \multicolumn{11}{|l|}{ Oilseed crops ${ }^{\mathrm{y}}$} \\
\hline BE0114 & $\mathrm{C}$ & Saphir & Spain & MBG & 0 & 95 & $7-9$ & 8.8 & $1-7$ & 2.5 \\
\hline BE0052 & $\mathrm{C}$ & Valle del Oro & Spain & MBG & 0 & 47 & $6-9$ & 8.6 & $2-8$ & 3.9 \\
\hline BE0059 & $\mathrm{AC}$ & Petranova & Germany & CGN & 0 & 56 & $7-9$ & 8.6 & $1-9$ & 4.1 \\
\hline MBG-BRS0392 & $\mathrm{L}$ & Kapusta zepak & Poland & MBG & 0 & 50 & 9 & 9.0 & $2-9$ & 4.8 \\
\hline BE0115 & $\mathrm{C}$ & Jura & Spain & MBG & 0 & 9 & $7-9$ & 8.7 & $3-9$ & 6.8 \\
\hline BE0068 & $\mathrm{AC}$ & Belinda & The Netherlands & CGN & 0 & 20 & $7-9$ & 8.2 & $2-9$ & 7.1 \\
\hline BE0049 & $\mathrm{C}$ & Express & Germany & MBG & 0 & 13 & $7-9$ & 8.4 & $2-9$ & 7.6 \\
\hline BE0050 & $\mathrm{C}$ & Quest & Canada & MBG & 0 & 0 & $8-9$ & 8.9 & $8-9$ & 8.9 \\
\hline BE0136 & $\mathrm{C}$ & Topas & Sweden & MBG & 0 & 0 & 9 & 9.0 & 9 & 9.0 \\
\hline Mean & $\ldots$ & $\ldots$ & $\ldots$ & $\ldots$ & $\ldots$ & $\ldots$ & $\ldots$ & 8.7 & $\ldots$ & 6.1 \\
\hline $\mathrm{CV}$ & $\ldots$ & $\ldots$ & $\ldots$ & $\ldots$ & $\ldots$ & $\ldots$ & $\ldots$ & 7.6 & $\ldots$ & 29.0 \\
\hline LSD (0.05) & $\ldots$ & $\ldots$ & $\ldots$ & $\ldots$ & $\ldots$ & $\ldots$ & $\ldots$ & 0.5 & $\ldots$ & 1.3 \\
\hline \multicolumn{11}{|c|}{ Root vegetable crops $^{\mathrm{z}}$} \\
\hline $\mathrm{BE} 0125$ & $\mathrm{AC}$ & York & Germany & CGN & 0 & 41 & $7-9$ & 8.3 & $1-9$ & 4.8 \\
\hline BE0064 & $\mathrm{AC}$ & Friese Gele & The Netherlands & $\mathrm{CGN}$ & 0 & 33 & $5-9$ & 7.1 & $2-9$ & 5.3 \\
\hline BE0001 & $\mathrm{C}$ & Colinabo Rocalba & Spain & MBG & 0 & 6 & $7-9$ & 8.6 & $2-9$ & 7.4 \\
\hline \multirow[t]{2}{*}{ BE0124 } & $\mathrm{AC}$ & York/Wilhelms-burger & & & & & & & & \\
\hline & & Hybrid & Germany & CGN & 0 & 0 & $7-9$ & 8.3 & $7-9$ & 8.4 \\
\hline BE0058 & $\mathrm{AC}$ & Lollo & Germany & CGN & 0 & 0 & $7-9$ & 8.7 & $7-9$ & 8.5 \\
\hline BE0126 & $\mathrm{AC}$ & - & New Zealand & $\mathrm{CGN}$ & 0 & 0 & $7-9$ & 8.8 & $7-9$ & 8.8 \\
\hline \multirow[t]{2}{*}{ MBG-BRS0023 } & $\mathrm{C}$ & Colinabo amarillo cuello & & & & & & & & \\
\hline & & verde & Spain & $\mathrm{MBG}$ & 0 & 0 & 9 & 9.0 & $7-9$ & 8.8 \\
\hline $\begin{array}{l}\text { BE0127 } \\
\text { BE0123 }\end{array}$ & $\begin{array}{l}\mathrm{AC} \\
\mathrm{L}\end{array}$ & $\begin{array}{l}\text { Hollandse Gele Roodkop } \\
\text { Nabo Murcia }\end{array}$ & $\begin{array}{l}\text { The Netherlands } \\
\text { Spain }\end{array}$ & $\begin{array}{l}\text { CGN } \\
\text { COMAV }\end{array}$ & $\begin{array}{l}0 \\
0\end{array}$ & $\begin{array}{l}0 \\
0\end{array}$ & $\begin{array}{l}7-9 \\
9\end{array}$ & $\begin{array}{l}8.8 \\
9.0\end{array}$ & $\begin{array}{l}7-9 \\
9\end{array}$ & $\begin{array}{l}8.9 \\
9.0\end{array}$ \\
\hline Mean & $\ldots$ & $\ldots$ & $\ldots$ & $\ldots$ & $\ldots$ & $\ldots$ & $\ldots$ & 8.6 & $\ldots$ & 8.0 \\
\hline $\mathrm{CV}$ & $\ldots$ & $\ldots$ & $\ldots$ & $\ldots$ & $\ldots$ & $\ldots$ & $\ldots$ & 7.7 & $\ldots$ & 17.9 \\
\hline LSD (0.05) & $\ldots$ & $\ldots$ & $\ldots$ & $\ldots$ & $\ldots$ & $\ldots$ & $\ldots$ & 0.5 & $\ldots$ & 1.0 \\
\hline LSD all (0.05) & $\ldots$ & $\ldots$ & $\ldots$ & $\ldots$ & $\ldots$ & $\ldots$ & $\ldots$ & 0.5 & $\ldots$ & 1.1 \\
\hline
\end{tabular}


were resistant to race 4, showing disease scores of 3.2. Again, the resistance test in these genotypes showed a variable reaction, including both resistant and susceptible plants. In the oleifera group, only the commercial rapeseed cv. Saphir was highly resistant to race 4 (95\% of resistant plants showing a disease score of 2 or 3 ). No resistance to race 4 was found in the napobrassica group, even though most accessions evaluated were improved cultivars. The only exceptions were Friese Gele and York, which exhibited moderate resistance, with a mean disease score around 5 in the 1to- 9 rating scale (Table 2).

Races 1 and 4. Some accessions showed plants with either resistance or partial resistance to races 1 and 4. Among them, Russian kale had two plants with resistance to races 1 and 4 and another two plants partially resistant to both races. Other promising cultivars were Friese Gele (from napobrassica group), which showed three plants with partial resistance to race 1 and resistance to race 4; Ragged Jack kale (from napus group), which presented two plants with partial resistance to race 1 and resistance to race 4; and Valle del Oro (a winter oilseed rape from Spain, included in the oleifera group), which had one plant with partial resistance for both races. Nabicol accessions MBG-BRS0037, MBG-BRS0041, and MBG-BRS0131, belonging to the pabularia group, included some plants with partial resistance to race 1 and both resistance and partial resistance to race 4 . In this case, race-nonspecific resistance could be involved and must be further studied.

\section{Discussion}

Black rot disease is particularly serious in leafy-green vegetable crops used for human consumption and belonging to the pabularia group. It would seem reasonable that this group showed the highest level of resistance because any lesion on the leaves is not acceptable. When the edible parts of the plant are seed or roots, as for accessions in the oleifera and napobrassica groups, this disease will probably not affect the marketable yield. However, it should be noted that black rot is an important constraint for these crops in early stages of the plant development. Infected young seedlings can show dwarfing, erratic growth, and one-sided biased, withered leaves (1). Tissue infected with $X$. campestris pv. campestris can serve as a point of entry for other fungal and bacterial organisms, further limiting seed and root yields.

No race-specific resistance to race 1 was found in the accessions evaluated. Even though strong and uniform resistance for race 1 was not found, the finding of variable reaction for resistance in some accessions is very relevant because resistance to race 1 is very unusual. As was already reported in several works $(23,25)$, resistance to race 1 is very uncommon and has been found only in Brassica crops carrying the B genome (B. carinata, $B$. juncea, and $B$. nigra). These accessions must be further evaluated to find resistant plants that can be used in breeding programs when resistance could be fixed and available (i.e., through doubled-haploids techniques).
A high degree of variability for resistance was found within each accession, producing a high proportion of variable reactions. A similar result was found by Westman et al. (33), highlighting the variability for resistance to $X$. campestris pv. campestris found in $B$. nigra genotypes. Taylor et al. (25) also found a high intravarietal variability for resistance to $X$. campestris pv. campestris in different $B$. oleracea crops. As was discussed by Vicente et al. (31), extreme reactions for resistance to $X$. campestris pv. campestris within an accession could be explained when resistance is controlled by a single gene or if a mixture of genotypes within a cultivar occurs.

According to Vicente (30), who studied the identification and distribution of $X$. campestris pv. campestris races in Portugal, it could be possible to find race 4 in northwestern Spain, where most of the pabularia accessions included in this study were collected. Local vegetable crops such as nabicol have been sown for generations in the same location, favoring disease. The high level of resistance to $X$. campestris pv. campestris race 4 found in most of the nabicol landraces could be partly explained by the selection practiced by growers throughout the years, rejecting abnormal and diseased plants for both human consumption and seed production.

Most of the B. napus commercial and improved cultivars evaluated in this work belong to the oleifera, napobrassica, and napus groups. The low resistance level to both races found in B. napus commercial and improved cultivars from these three groups suggests that breeding for $X$. campestris pv. campestris resistance has not been a priority in those crops. Breeding in B. napus has been mainly focused on oilseed quality and fresh yield but not on disease resistance. Furthermore, breeding for $X$. campestris pv. campestris resistance in Brassica crops has had limited success, mainly due to the low number of available sources of resistance and the difficulties in transferring that resistance among Brassica spp. (26). However, resistance to race 4 found in commercial leafy vegetable $B$. napus crops from the pabularia group suggests that some type of indirect breeding for resistance to $X$. campestris pv. campestris could have happened, because damaged leaves are not allowed in the fresh market.

In the gene-for-gene model established by Vicente et al. (31), resistance to race 1 is conferred by the $\mathrm{R} 1$ gene, possibly originating from the $\mathrm{B}$ genome of $B$. nigra, and resistance to race 4 is conferred by the R4 gene, possibly originating from the A genome of B. rapa. This is consistent with the hypothesis that, in our B. napus accessions (AC genome), the amphidiploid species derived from interspecific hybridization between $B$. oleracea ( $\mathrm{C}$ genome) and $B$. rapa (A genome), resistance to race 4 is present and resistance to race 1 is practically absent (mean disease scores to races 4 and 1 were 5.8 and 8.6, respectively). In the model proposed by Vicente et al. (31) and Taylor et al. (25), in the AC genome of B. napus, the $\mathrm{R} 1$ gene conferring resistance to race 1 is not present. This supports the theory that the resistance found in some accessions could be due to a race-nonspecific resistance. However, inoculation with

Table 3. Frequency of resistant accessions and mean disease score for Xanthomonas campestris pv. campestris races 1 and 4 of 76 Brassica napus accessions included in this study and grouped according to their main end use

\begin{tabular}{|c|c|c|c|c|c|}
\hline \multirow[b]{2}{*}{ Use } & \multirow[b]{2}{*}{ Total no. ${ }^{x}$} & \multicolumn{2}{|c|}{ No. of resistant accessions ${ }^{v}$} & \multicolumn{2}{|c|}{ Disease score $(1-9)^{\mathrm{w}}$} \\
\hline & & Race 1 & Race 4 & Race 1 & Race 4 \\
\hline Forage crops (napus group) & 10 & 0 & 8 & $8.4 \mathrm{~b}$ & $5.4 \mathrm{c}$ \\
\hline Leafy vegetable crops (pabularia group) & 48 & 1 & 42 & $8.7 \mathrm{a}$ & $3.4 \mathrm{~d}$ \\
\hline Oilseed crops (oleifera group) & 9 & 0 & 6 & $8.7 \mathrm{a}$ & $6.1 \mathrm{~b}$ \\
\hline Root vegetable crops (napobrassica group) & 9 & 0 & 2 & $8.6 \mathrm{a}$ & $8.0 \mathrm{a}$ \\
\hline Mean & $\ldots$ & $\ldots$ & $\ldots$ & 8.6 & 4.6 \\
\hline $\mathrm{CV}^{\mathrm{y}}$ & $\ldots$ & $\ldots$ & $\ldots$ & 7.9 & 35.0 \\
\hline $\operatorname{LSD}(0.05)^{\mathrm{z}}$ & $\ldots$ & $\ldots$ & $\ldots$ & 0.16 & 0.51 \\
\hline
\end{tabular}

${ }^{\mathrm{v}}$ Accessions including more than one resistant plant.

${ }^{\text {w }}$ Disease score based on a rating scale from 1 to 9 , where $1=$ plants without lesions and $9=$ severely diseased plants. Means in columns followed by the same letter are not significantly different at $P \leq 0.05$.

${ }^{x}$ Total number of accessions.

${ }^{\mathrm{y}}$ Coefficient of variation.

${ }^{\mathrm{z}}$ Least significant difference. 
other $X$. campestris pv. campestris races, particularly race 6 , must be done to verify this finding. In a recent study performed by Soengas et al. (22), based on morphologic and agronomic traits of different $B$. napus crops, the Russian kale landrace was grouped apart from other accessions. This cultivar was completely different from other $B$. napus accessions evaluated, showcasing characteristic leaf morphology with divided and wavy leaf margins. A relationship between leaf morphological attributes and X. campestris pv. campestris resistance must be further investigated.

A comparison of black rot resistance among crops within $B$. napus species was achieved for the first time, emphasizing the search for sources of resistance in leafy vegetable crops. In this study, we suggest that different selection criteria applied on $B$. napus crops according to their use could have led to an indirect selection for resistance to $X$. campestris pv. campestris. Because resistance to race 1 seems to be absent in the B. napus genome, the moderate levels of resistance found in some cultivars turn them into promising sources of resistance. Because the inoculation method (juvenile wound inoculation by using a concentrated bacterial suspension) and conditions (favorable greenhouse environment for the $X$. campestris pv. campestris development) used in this study were severe, higher levels of resistance can be expected in the field in those moderately resistant cultivars (7). Resistance to race 4 was found in the pabularia, napus, and oleifera groups, mainly in local nabicol accessions belonging to the first group. Thus, the potential value of nabicol accessions to improve resistance to race 4 in Brassica crops is noteworthy, especially in those areas where vegetable $B$. napus crops are grown (i.e., the Atlantic coast of Europe) and black rot causes leaf damage, consequently decreasing crop quality and value. These accessions could be directly used in breeding programs to obtain resistant cultivars or used as donors of race-specific resistance. Related Brassica spp. have been used to introgress disease resistance and other agronomic and horticultural traits into $B$. oleracea, even though chromosome numbers differ, by using different approaches such as protoplast fusion (9) or embryo rescue (27). This is due to the high tolerance of aneuploidy and common ancestry in diploids. Resistant nabicol accessions could be used to transfer resistance to race 4 into other vegetable Brassica crops that share a common genome with $B$. napus, where resistance to races 1 and 4 of $X$. campestris pv. campestris is not present. Additionally, if new resistance genes are implicated in the response to black rot in our materials that differ from those detected by Griffiths et al. (6), then efforts can be made to complement and pyramid Brassica sources of black rot resistance to obtain durable resistance to $X$. campestris pv. campestris. Further theoretical and practical applications with these materials could focus on inheritance and mechanism of resistance studies and development of molecular markers for black rot resistance.

\section{Acknowledgments}

Research was supported by the project AGL2009-09922 and Excma. Diputación Provincial de Pontevedra. We thank J. Vicente for supplying the bacterial isolates and susceptible and resistant checks.

\section{Literature Cited}

1. Agrios, G. N. 2005. Plant Pathology. Elsevier Academic Press, San Diego, CA.

2. Bain, D. C. 1952. Reaction of Brassica seedlings to black rot. Phytopathology 42:497-500.

3. Camargo, L. E. A., Williams, P. H., and Osborn, T. C. 1995. Mapping of quantitative trait loci controlling resistance of Brassica oleracea to Xanthomonas campestris pv. campestris in the field and greenhouse. Phytopathology 85:1296-1300.

4. Dickson, M. D., and Hunter, J. E. 1987. Inheritance of resistance in cabbage seedlings to black rot. HortScience 22:108-109.

5. Fargier, E., and Manceau, C. 2007. Pathogenicity assays restrict the species Xanthomonas campestris into three pathovars and reveal nine races within X. campestris pv. campestris. Plant Pathol. 56:805-818.

6. Griffiths, P. D., Marek, L. F., and Robertson, L. D. 2009. Identification of crucifer accessions from the NC-7 and NE-9 Plant Introduction Collections that are resistant to black rot (Xanthomonas campestris pv. campestris) races 1 and 4. HortScience 44:284-288.

7. Griffiths, P. D., and Roe, C. 2005. Response of Brassica oleracea var. capitata to wound and spray inoculations with Xanthomonas campestris pv. campestris. HortScience 40:47-49.
8. Guo, H., Dickson, M. H., and Hunter, J. E. 1991. Brassica napus sources of resistance to black rot in crucifers and inheritance of resistance HortScience 26:1545-1547.

9. Hansen, L. N., and Earle, E. D. 1995. Transfer of resistance to Xanthomonas campestris pv. campestris into Brassica oleracea $\mathrm{L}$. by protoplast fusion. Theor. Appl. Genet. 91:1293-1300.

10. Hunter, J. E., Dickson, M. H., and Ludwig, J. W. 1987. Source of resistance to black rot of cabbage expressed in seedlings and adult plants. Plant Dis. 71:263-266.

11. Ignatov, A., Kuginuki, Y., and Hida, K. 1998. Race-specific reaction of resistance to black rot in Brassica oleracea. Eur. J. Plant Pathol. 104:821-827.

12. Jensen, B. D., Massomo, S. M. S., Swai, I. S., Hockenhull, J., and Andersen, S. B. 2005. Field evaluation for resistance to the black rot pathogen Xanthomonas campestris pv. campestris in cabbage (Brassica oleracea). Eur. J. Plant Pathol. 113:297-308.

13. Kamoun, S., Kamdar, H. V., Tola, E., and Kado, C. I. 1992. Incompatible interactions between crucifers and Xanthomonas campestris involve a vascular hypersensitive response: role of the $h r p X$ locus. Mol. Plant-Microbe Interact. 5:22-33.

14. Lema, M., Soengas, P., Francisco, M., and Velasco, P. 2008. Diseases affecting Brassica crops in Northwestern of Spain. Cruciferae Newsl. 27:24-26.

15. Lema-Marquez, M., Teran, H., and Singh, S. P. 2007. Selecting common bean with genes of different evolutionary origins for resistance to Xanthomonas campestris pv. phaseoli. Crop Sci. 47:1367-1374.

16. Massomo, S. M. S., Mabagala, R. B., Swai, I. S., Hockenhull, J., and Mortensen, C. N. 2004. Evaluation of varietal resistance in cabbage against the black rot pathogen, Xanthomonas campestris pv. campestris in Tanzania. Crop Prot. 23:315-325.

17. Nogueira, A. P., and Dias, J. S. 1997. Screening of a Brassica rapa collection with two Portuguese isolates of Xanthomonas campestris pv. campestris. Page 211 in: Second ISHS Symposium on Brassicas, 10th Crucifer Genetics Workshop. G. G. Thomas and A. A. Monteiro, eds. Rennes.

18. Ortega, A. M., and López, M. M. 1990. Identificación de Xanthomonas campestris pv. campestris (Pammel) Dowson en distintas especies de Brassica. Pages 199-204 in: Proc. V Congr. Soc. Española Fitopatol. Badajoz.

19. Ribeiro, B., and Dias, J. S. 1997. Screening of a Brassica napus collection with two Portuguese isolates of Xanthomonas campestris pv. campestris. Page 210 in: Second ISHS Symp. Brassicas, 10th Crucifer Genet. Workshop. G. G. Thomas and A. A. Monteiro, eds. Rennes.

20. Rodríguez, V. M., Cartea, M. E., Padilla, G., Velasco, P., and Ordás, A. 2005. The nabicol: a horticultural crop in northwestern Spain. Euphytica 142:237-246.

21. SAS Institute Inc. 2002. SAS OnlineDoc, Version 9.1. SAS Institute Inc., Cary, NC.

22. Soengas, P., Cartea, M. E., Velasco, P., Padilla, G., and Ordás, A. 2008 Morphologic and agronomic diversity of Brassica napus crops. J. Am. Soc. Hortic. Sci. 133:48-54.

23. Soengas, P., Hand, P., Vicente, J. G., Pole, J. M., and Pink, D. A. C. 2007. Identification of quantitative trait loci for resistance to Xanthomonas campestris pv. campestris in Brassica rapa. Theor. Appl. Genet. 114:637-645.

24. Steel, R. G. D., Torrie, J. H., and Dickey, D. A. 1997. Principles and Procedures of Statistics. A Biometrical Approach. McGraw-Hill, New York.

25. Taylor, J. D., Conway, J., Roberts, S. J., Astley, D., and Vicente, J. G. 2002. Sources and origin of resistance to Xanthomonas campestris pv. campestris in Brassica genomes. Phytopathology 92:105-111.

26. Tonguc, M., Earle, E. D., and Griffiths, P. D. 2003. Segregation distortion of Brassica carinata derived black rot resistance in Brassica oleracea. Euphytica 134:269-276.

27. Tonguc, M., and Griffiths, P. D. 2004. Development of black rot resistant interspecific hybrids between Brassica oleracea L. cultivars and Brassica accession A 19182, using embryo rescue. Euphytica 136:313-318.

28. Tonguc, M., and Griffiths, P. D. 2004. Evaluation of Brassica carinata accessions for resistance to black rot (Xanthomonas campestris pv. campestris). HortScience 39:952-954

29. Urquijo, P., Sardiña, J. R., and Santaolalla, G., eds. 1970. Patología Vegetal Agrícola. Mundi-Prensa, Madrid.

30. Vicente, J. G. 2004. A Podridão Negra das Cruciferas. Centro Operativo e Tecnológico Hortofrutícola Nacional, Alcobaça.

31. Vicente, J. G., Conway, J., Roberts, S. J., and Taylor, J. D. 2001. Identification and origin of Xanthomonas campestris pv. campestris races and related pathovars. Phytopathology 91:492-499.

32. Vicente, J. G., Taylor, J. D., Sharpe, A. G., Parkin, I. A. P., Lydiate, D. J., and King, G. J. 2002. Inheritance of race-specific resistance to Xanthomonas campestris pv. campestris in Brassica genomes. Phytopathology 92:1134-1141.

33. Westman, A. L., Kresovich, S., and Dickson, M. H. 1999. Regional variation in Brassica nigra and other weedy crucifers for disease reaction to Alternaria brassicicola and Xanthomonas campestris pv. campestris. Euphytica 106:253-259.

34. Williams, P. H. 1980. Black rot: a continuing threat to world crucifers. Plant Dis. 64:736-742.

35. Williams, P. H.2007. Black rot. Pages 60-62 in: Compendium of Brassica Diseases. S. R. Rimmer, V. I. Shattuck, and L. Buchwaldt, eds. American Phytopathological Society, St. Paul, MN 